\title{
On the influence of model nonlinearity and localization on ensemble Kalman smoothing
}

\author{
Lars Nerger $^{1 *}$, Svenja Schulte ${ }^{2}$, and Angelika Bunse-Gerstner ${ }^{2}$ \\ ${ }^{1}$ Alfred Wegener Institute, Bremerhaven, Germany, ${ }^{2}$ University of Bremen, Germany \\ ${ }^{*}$ Correspondence to: Lars Nerger, Alfred Wegener Institute, Am Handelshafen 12, D-27570 Bremerhaven, Germany. \\ E-mail: Lars.Nerger@awi.de
}

Ensemble-based Kalman smoother algorithms extend ensemble Kalman filters to reduce the estimation error of past model states utilizing observational information from the future. Like the filters they extend, current smoothing algorithms are optimal only for linear models. However, the ensemble methods are typically applied with high-dimensional nonlinear models, which also require the application of localization in the data assimilation. In this paper, the influence of the model nonlinearity and of the application of localization on the smoother performance is studied. Numerical experiments show that the observational information can be successfully utilized over smoothing lags several times the error doubling time of the model. Localization limits the smoother lag by spatial decorrelation. However, if the localization is well tuned, the usable lag of the smoother, and hence the usable amount of observational information, is maximized. The localization reduces the estimation errors of the smoother even more than those of the filter. As the smoother reuses the transformation matrix of the filter, it profits stronger from increases of the ensemble size than the filter. With respect to inflation and localization, the experiments also show that the same configuration that yields the smallest estimation errors for the filter without smoothing also results in the smallest errors of the smoothed states. Thus, smoothing only adds the lag as a further tunable parameter. 


\section{Introduction}

Data assimilation with ensemble-based filter algorithms is performed to obtain estimates, e.g. of the model state, by combining the model prediction and observational data that are available until the time when the filter analysis step is computed. The sequential data assimilation methods with alternating forecast phases and analysis steps provide a trajectory of state estimates, each using the observations available until the time of the analysis. Reanalysis applications estimate the state over a time period in the past. Thus, observations for the full time interval are available at the time when the reanalysis is computed. For this application, a retrospective analysis is of interest in which also future observations during the time interval are used for the estimation of past states. Ideally, one can utilize the observational information from the full reanalysis time interval to estimate the state trajectory during this interval. This application is called smoothing.

Smoothing can be performed with ensemble-based algorithms. A first ensemble smoother was derived by van Leeuwen and Evensen (1996). This formulation exhibited a limited performance with nonlinear models, which was attributed to the fact that the method did not perform sequential updates but a backward smoothing operation of the full time interval using ensemble forecasts over the same time interval (Evensen and van Leeuwen 2000). The performance of ensemble smoothers was improved by a sequential formulation termed Ensemble Kalman Smoother (EnKS, Evensen and van Leeuwen 2000; van Leeuwen 2001). Evensen (2003) discussed that smoothing can be performed as a computationally cheap extension of the Ensemble Kalman Filter because the smoother computes a state correction at a previous time by combining the ensemble members at that time with weights given from the filter analysis at the current time. The computing cost of the smoother has been further discussed by Ravela and McLaughlin (2007), where a cost-reduction is proposed that avoids the recursive update at previous times. A review of different smoother formulations was conducted by Cosme et al. (2012). Ensemble-smoothers have been applied to complex models, for example, for state estimation in the North Atlantic Ocean (Brusdal et al. 2003), in an idealized atmospheric model (Khare et al. 2008), and in an idealized configuration of a high-resolution ocean model (Cosme et al. 2010).

Khare et al. (2008) examined the performance of ensemble smoothers in relation to the ensemble size and the accuracy of the observations. Their experiments showed that localization resulted in reduced errors in the smoothed states. Next to an idealized atmospheric model, the 40dimensional model by Lorenz (1996) was used to study the smoother performance. Khare et al. (2008) pointed to the relevance of sampling errors, e.g. due to small ensembles, in limiting the time lag over which a smoother can be applied. The lag at which the smallest errors were obtained was limited by spurious correlations caused by sampling errors. The effect was also discussed by Cosme et al. (2010) in case of a high-resolution ocean model. While the models used by Khare et al. (2008) and Cosme et al. (2010) were nonlinear, the influence of the nonlinearity was not examined. However, Khare et al. (2008) noted that the time evolution of ensemble perturbations is better approximated by linearized dynamics with a smaller ensemble spread. Further, Cosme et al. (2010) mention the expectation that nonlinearity will lead to a decorrelation of the different times involved in the smoothing and hence a convergence of the smoothed state estimate.

Ensemble Kalman smoothers, as other linear smoothers, are only optimal for linear dynamics (Cohn et al. 1994). In this case, the ensemble smoother solution becomes equivalent to the estimates obtained by 4dimensional variational assimilation methods (Fisher et al. 2005) if the initial ensemble represents the same covariance matrix as used in the variational method. While the smoother is suboptimal for nonlinear systems, the previous studies demonstrated that a smoother can still 
improve the state estimates. However, it is still unknown to which extent the nonlinearity influences the smoother.

To this end, this study focuses on two aspects: First, the influence of model nonlinearity on the smoother performance is assessed for an example application. Second, as high-dimensional models typically require the application of localization, the effect of the localization on the smoother performance is examined. In section 2 , an ensemble smoother is formulated as the extension of an ensemble square-root Kalman filter. The smoother is then applied in twin assimilation experiments with the 40dimensional Lorenz-96 model in section 3. By varying the forcing parameter of this low-dimensional model, the nonlinearity of the model is controlled and the smoother performance is studied in dependence of the nonlinearity. The influence of localization is assessed by varying the localization radius and the ensemble size. Subsequently, the application of the smoother to a realistic large scale ocean circulation model is discussed in section 4 . The findings of the experiments are summarized and conclusions are drawn in section 5 .

\section{Filter and Smoother Algorithms}

A smoother algorithm can be formulated as an extension of an ensemble-based filter. In this study, the smoother is discussed in the context of the Error Subspace Transform Kalman Filter (ESTKF, Nerger et al. 2012b). However, due to the similarity to other algorithms like the Ensemble Transform Kalman Filter (ETKF, Bishop et al. 2001), LETKF (Hunt et al. 2007), or the SEIK filter (Pham 2001), the application in these filters is analogous. Also, the smoother extension of the SEEK filter (Cosme et al. 2010) is similar.

\subsection{Error Subspace Transform Kalman Filter (ESTKF)}

In all ensemble-based Kalman filters, the state vector $\mathbf{x}_{l}$ of size $n$ and the corresponding error covariance matrix $\mathbf{P}_{l}$ represent the state of a physical system and its error estimate at time $t_{l}$. These quantities are represented by an ensemble of $m$ vectors $\mathbf{x}^{(j)}, j=1, \ldots, m$, of model state realizations. The state estimate is given by the ensemble mean

$$
\overline{\mathbf{x}}_{l}:=\frac{1}{m} \sum_{j=1}^{m} \mathbf{x}_{l}^{(j)} .
$$

Using the matrix of ensemble perturbations

$$
\mathbf{X}_{l}^{\prime}:=\mathbf{X}_{l}-\overline{\mathbf{X}_{l}}
$$

with the ensemble matrix $\mathbf{X}_{l}:=\left[\mathbf{x}_{l}^{(1)}, \ldots, \mathbf{x}_{l}^{(m)}\right]$, shortly referred to as the ensemble, and $\overline{\mathbf{X}_{l}}:=\left[\overline{\mathbf{x}_{l}}, \ldots, \overline{\mathbf{x}_{l}}\right], \mathbf{P}_{l}$ is given as the ensemble covariance matrix

$$
\mathbf{P}_{l}:=\frac{1}{m-1} \mathbf{X}_{l}^{\prime}\left(\mathbf{X}_{l}^{\prime}\right)^{T}
$$

A forecast ensemble $\mathbf{X}_{k}^{f}$ at the observation time $t_{k}$ is computed by integrating the state ensemble using the numerical model. The vector of observations $\mathbf{y}_{k}$ of size $p$ is related to the model state by $\mathbf{y}_{k}=\mathbf{H}_{k}\left(\mathbf{x}_{k}^{f}\right)+\boldsymbol{\epsilon}_{k}$, where $\mathbf{H}$ is the observation operator. The vector of observation errors, $\epsilon_{k}$, is assumed to be a white Gaussian distributed random process with covariance matrix $\mathbf{R}$.

For the ESTKF, the forecast covariance matrix $\mathbf{P}_{k}^{f}$ is written as

$$
\mathbf{P}_{k}^{f}=\frac{1}{m-1} \mathbf{L}_{k} \mathbf{L}_{k}^{T}
$$

where $\mathbf{L}_{k}$ is a matrix of size $n \times(m-1)$ defined by

$$
\mathbf{L}_{k}:=\mathbf{X}_{k}^{f} \mathbf{T}
$$

The matrix $\mathbf{T}$ projects the ensemble matrix onto the error subspace represented by the ensemble. It has size $m \times$ $(m-1)$ and is defined by:

$$
\mathbf{T}_{j, i}:=\left\{\begin{aligned}
1-\frac{1}{m} \frac{1}{\frac{1}{\sqrt{m}}+1} & \text { for } i=j, j<m \\
-\frac{1}{m} \frac{1}{\frac{1}{\sqrt{m}}+1} & \text { for } i \neq j, j<m \\
-\frac{1}{\sqrt{m}} & \text { for } j=m
\end{aligned}\right.
$$

Geometrically, $\mathbf{T}$ is the Householder matrix associated with the vector $m^{-1 / 2}(1, \ldots, 1)^{T}$. 
For the analysis, one defines a transform matrix $\mathbf{A}_{k}$ of size $(m-1) \times(m-1)$ by

$$
\mathbf{A}_{k}^{-1}:=\rho(m-1) \mathbf{I}+\left(\mathbf{H}_{k} \mathbf{L}_{k}\right)^{T} \mathbf{R}_{k}^{-1} \mathbf{H}_{k} \mathbf{L}_{k}
$$

where $\mathbf{I}$ is the identity and $\rho$ with $0<\rho \leq 1$ is the "forgetting factor" that is used to implicitly inflate the forecast error covariance estimate. Using $\mathbf{A}_{k}$, the analysis covariance matrix is given by

$$
\mathbf{P}_{k}^{a}=\mathbf{L}_{k} \mathbf{A}_{k} \mathbf{L}_{k}^{T}
$$

The analysis ensemble is computed as a correction of the ensemble mean and a transformation of the ensemble perturbations. The analysis state estimate is computed from the forecast using a combination of the columns of the matrix $\mathbf{L}_{k}$ by

$$
\overline{\mathbf{x}_{k}^{a}}=\overline{\mathbf{x}_{k}^{f}}+\mathbf{L}_{k} \overline{\mathbf{w}_{k}}
$$

with the weight vector $\overline{\mathbf{w}_{k}}$ of size $m-1$ given by

$$
\overline{\mathbf{w}_{k}}:=\mathbf{A}_{k}\left(\mathbf{H}_{k} \mathbf{L}_{k}\right)^{T} \mathbf{R}_{k}^{-1}\left(\mathbf{y}_{k}-\mathbf{H}_{k} \overline{\mathbf{x}_{k}^{f}}\right)
$$

The ensemble is now transformed as

$$
\mathbf{X}_{k}^{a}=\overline{\mathbf{X}_{k}^{a}}+\mathbf{L}_{k} \mathbf{W}_{k}
$$

where the weight matrix $\mathbf{W}_{k}$ is defined by

$$
\mathbf{W}_{k}:=\sqrt{m-1} \mathbf{C}_{k} \mathbf{T}^{T} \boldsymbol{\Lambda}
$$

Here, $\mathbf{C}_{k}$ is the symmetric square root of $\mathbf{A}_{k}$ that is computed from the singular value decomposition $\mathbf{U}_{k} \mathbf{S}_{k} \mathbf{V}_{k}=\mathbf{A}_{k}^{-1}$ such that $\mathbf{C}_{k}=\mathbf{U}_{k} \mathbf{S}_{k}^{-1 / 2} \mathbf{U}_{k}^{T}$. The matrix $\boldsymbol{\Lambda}$ of size $m \times m$ is an arbitrary orthogonal matrix or the identity. The vector $(1, \ldots, 1)^{T}$ has to be an eigenvector of $\boldsymbol{\Lambda}$ to ensure that the ensemble mean is preserved.

For efficiency, the computation of the analysis state estimate, Eq. (9), and the transformation of the ensemble perturbations, Eq. (11), can be combined into a single transformation as

$$
\mathbf{X}_{k}^{a}=\overline{\mathbf{X}_{k}^{f}}+\mathbf{L}\left(\overline{\mathbf{W}_{k}}+\mathbf{W}_{k}\right)
$$

with $\overline{\mathbf{W}_{k}}:=\left[\overline{\mathbf{w}_{k}}, \ldots, \overline{\mathbf{w}_{k}}\right]$. In addition, the term $\mathbf{H}_{k} \mathbf{L}_{k}$ in equations (7) and (10) is typically computed as $\left(\mathbf{H}_{k} \mathbf{X}_{k}^{f}\right) \mathbf{T}$. Thus, $\mathbf{T}$ operates on the $p \times m$ matrix $\mathbf{H}_{k} \mathbf{X}_{k}^{f}$, while $\mathbf{H}_{k}$ operates on each ensemble state. Further, the matrix $\mathbf{L}$ in Eq. (13) can be replaced by its definition $\mathbf{X}_{k}^{f} \mathbf{T}$. Then, the matrix $\mathbf{T}$ can be applied from the left to the small $m \times m$ matrix $\overline{\mathbf{W}_{k}}+\mathbf{W}_{k}$.

\subsection{Localization}

Localization allows ensemble Kalman filters to operate successfully with small ensembles in high-dimensional models. For the ESTKF, the domain localization of the SEIK filter (Nerger et al. 2006) with observation localization (Hunt et al. 2007) is used. Here, a short review for the local ESTKF is provided.

For the domain localization, the analysis and the ensemble transformation of the ESTKF are performed in a loop through disjoint local analysis domains. In the simplest case, each single grid point is updated independently. For each local analysis domain, the observations are weighted by their distance from this domain by multiplying the matrix $\mathbf{R}^{-1}$ element-wise with a localization matrix $\tilde{\mathbf{D}}$. $\tilde{\mathbf{D}}$ is constructed from a correlation function with compact support such that observations beyond a certain distance obtain zero weight. These observations can be neglected for the local analysis update. The local analysis domain will be denoted by the subscript $\sigma$. The domain of the corresponding observations of non-zero weight is denoted by $\delta$. Dropping the time index $k$ for compactness, the ESTKF with localization can now be written as

$$
\mathbf{X}_{\sigma}^{a}=\overline{\mathbf{X}_{\sigma}^{f}}+\mathbf{L}_{\sigma}\left(\left[\overline{\mathbf{w}_{\delta}}, \ldots, \overline{\mathbf{w}_{\delta}}\right]+\mathbf{W}_{\delta}\right)
$$

$$
\overline{\mathbf{w}_{\delta}}=\mathbf{A}_{\delta}\left(\mathbf{H}_{\delta} \mathbf{L}\right)^{T}\left(\tilde{\mathbf{D}}_{\delta} \circ \mathbf{R}_{\delta}^{-1}\right)\left(\mathbf{y}_{\delta}^{o}-\mathbf{H}_{\delta} \overline{\mathbf{x}^{f}}\right),
$$




$$
\begin{gathered}
\mathbf{A}_{\delta}^{-1}=\rho_{\delta}(m-1) \mathbf{I}+\left(\mathbf{H}_{\delta} \mathbf{L}\right)^{T}\left(\tilde{\mathbf{D}}_{\delta} \circ \mathbf{R}_{\delta}^{-1}\right) \mathbf{H}_{\delta} \mathbf{L}, \\
\mathbf{W}_{\delta}=\sqrt{m-1} \mathbf{C}_{\delta} \mathbf{T}^{T} \boldsymbol{\Lambda} .
\end{gathered}
$$

Here, o denotes the element-wise product. The matrix $\mathbf{C}_{\delta}$ is the symmetric square root of $\mathbf{A}_{\delta}$.

In the numerical experiments, the matrix $\tilde{\mathbf{D}}_{\delta}$ is constructed using a 5 th order polynomial function (Eq. 4.10 of Gaspari and Cohn 1999), which mimics a Gaussian function but has compact support. The distance at which the function becomes zero defines the localization radius.

\subsection{The smoother extension ESTKS}

The smoother extension of the ESTKF is formulated analogously to the ensemble Kalman smoother (EnKS, Evensen 2003). The sequential smoother computes a state correction at an earlier time $t_{k-l}$ with lag $l$, utilizing the filter analysis update at time $t_{k}$.

For the smoother, the notation is extended according to the notation used in estimation theory (see, e.g., Cosme et al. 2010): A subscript $i \mid j$ is used, where $i$ refers to the time that is represented by the state vector and $j$ refers to the latest time for which observations are taken into account. Thus, the filter analysis state $\mathbf{x}_{k}^{a}$ is written as $\mathbf{x}_{k \mid k}^{a}$ and the forecast state $\mathbf{x}_{k}^{f}$ is denoted as $\mathbf{x}_{k \mid k-1}^{f}$. The superscripts $a$ and $f$ are redundant in this notation.

To formulate the smoother, the transformation equation (13) is first written as a product of the forecast ensemble with a weight matrix as

$$
\mathbf{X}_{k \mid k}^{a}=\mathbf{X}_{k \mid k-1}^{f} \mathbf{G}_{k}
$$

with

$$
\mathbf{G}_{k}:=\mathbf{1}_{(m)}+\mathbf{T}\left(\overline{\mathbf{W}_{k}}+\mathbf{W}_{k}\right) .
$$

Here the relation $\overline{\mathbf{X}}_{k \mid k-1}^{f}=\mathbf{X}_{k \mid k-1}^{f} \mathbf{1}_{(m)}$ is used with the matrix $\mathbf{1}_{(m)}$ that contains the value $m^{-1}$ in all entries. If no inflation is applied, the smoothed state ensemble at time $t_{k-1}$ taking into account the observations up to time $t_{k}$ is computed from the analysis state ensemble $\mathbf{X}_{k-1 \mid k-1}^{a}$ as

$$
\mathbf{X}_{k-1 \mid k}^{a}=\mathbf{X}_{k-1 \mid k-1}^{a} \mathbf{G}_{k} .
$$

The smoothing at time $t_{i}$ with $i=k-l$ by future observations at different analysis times is computed by multiplying $\mathbf{X}_{i \mid i}^{a}$ with the corresponding matrices $\mathbf{G}_{j}$ for all previous analysis times $t_{j}, i<j \leq k$. Thus, the smoothed state ensemble is given by

$$
\mathbf{X}_{i \mid k}^{a}=\mathbf{X}_{i \mid i}^{a} \prod_{j=i+1}^{k} \mathbf{G}_{j}
$$

Equations (18) to (21) are likewise valid for the global and local filter variants. Thus, $\mathbf{G}_{k}$ can be computed for the global analysis and then applied to all rows of the global matrix $\mathbf{X}_{i \mid j}^{a}$, or for the local weights of section 2.2 and applied to the ensemble of the corresponding local analysis domain $\sigma$. In the following sections, $\mathbf{X}_{i \mid k}^{a}$ will be referred to as the filter solution if $i=k$ and as the smoother solution in the case of $i<k$.

For the case that covariance inflation is applied, it has to be accounted for in the smoother calculations. As discussed by Cosme et al. (2010), the equation for the temporal cross-covariances does not contain a model-error term. Hence, an ensemble that was inflated by a forgetting factor has to be deflated for the application in the smoother step. For the ESTKS with inflation, one has to define the weight matrix for smoothing by

$$
\tilde{\mathbf{G}}_{k}:=\mathbf{1}_{(m)}+\rho \mathbf{T}\left(\overline{\mathbf{W}_{k}}+\mathbf{W}_{k}\right) .
$$

The application of $\rho$ in Eq. (22) removes the inflation from the weight matrices that has been introduced by Eq. (7)* For the smoother step, the deflated matrix $\tilde{\mathbf{G}}_{k}$ is used in Eq. (21), while for the filter $\mathbf{G}_{k}$ defined by Eq. (19) is used.

* The factor $\rho$ used here is distinct from the factor $\sqrt{\rho}$ used by Cosme et al. (2010). The difference is required because in the ESTKF the inflation is applied in the computation of the matrix $\mathbf{A}$ (Eq. 7), which is computationally cheaper than the direct inflation of the ensemble perturbations in Cosme et al. (2010) because of the smaller size of $\mathbf{A}$. 
The smoother can also be applied in the case that the matrix $\Lambda$ in Eq. (12) is a random matrix. This is due to the fact that the random transformation of a filter analysis at time $t_{k}$ is contained in the forecast and analysis ensembles at future times.

\subsection{Properties of the smoother with linear and nonlinear} systems

The ensemble smoothers like the ESTKS in section 2.3 are optimal for linear dynamical systems in the sense that the forecast of the smoothed state ensemble $\mathbf{X}_{i \mid k}^{a}$ with the linear model until the time $t_{k}$ results in a state ensemble that is identical to the analysis state ensemble $\mathbf{X}_{k \mid k}^{a}$. This property can be easily derived by applying the linear model operator $\mathbf{M}_{k, i}$ with $\mathbf{X}_{k, i}^{f}=\mathbf{M}_{k, i} \mathbf{X}_{i \mid i}^{a}$ and using Eq. (18) recursively. With Eq. (21) one obtains for $\rho=1$, i.e. no model errors,

$$
\mathbf{X}_{k \mid k}^{a}=\mathbf{M}_{k, i} \mathbf{X}_{i, i}^{a} \prod_{j=i+1}^{k} \mathbf{G}_{j}=\mathbf{M}_{k, i} \mathbf{X}_{i, k}^{a}
$$

If the model operator $\mathbf{M}_{k, i}$ is an orthogonal matrix, the forecast will preserve the ensemble variance. In this case, the smoothing will result in constant smoother errors over the full time interval $\left[t_{0}, t_{k}\right]$. Such an orthogonal matrix is, for example, given by the linear advection model used by Evensen (2004).

The formulation of the smoother used in section 2.3 hides the fact that the smoothing involves the crosscovariances between the analysis ensemble at the filter time and the ensemble at smoothing time. This can be seen from the alternative formulation for the state correction by smoothing that is used in the EnKS (see Evensen 2003):

$$
\overline{\mathbf{x}_{i \mid k}^{a}}=\overline{\mathbf{x}_{i \mid k-1}^{a}}+\mathbf{X}^{\prime a}{ }_{i, k-1}\left(\mathbf{X}_{k, k}^{\prime a}\right)^{T} \mathbf{E}
$$

with $\mathbf{E}$ given by

$$
\mathbf{E}:=\mathbf{H}_{k}^{T}\left(\mathbf{H}_{k} \mathbf{P}_{k}^{f} \mathbf{H}_{k}^{T}+\mathbf{R}_{k}^{-1}\right)^{-1}\left(\mathbf{y}_{k}-\mathbf{H}_{k} \overline{\mathbf{x}}_{k \mid k-1}\right)
$$

This alternative formulation shows that the smoothing will only have an effect if the ensembles at the filter and smoothing times are correlated.

The behavior of the smoother in the case a linear system with known model and observation error covariance matrices was examined by Cohn et al. (1994). In this situation, the smoother is guaranteed to reduce the error variance of the smoothed state estimate as long as the state errors at the times $i$ and $k$ are correlated. If the errors are uncorrelated, the smoothing has no effect. Cohn et al. (1994) also found that model errors lead to a faster convergence of the smoother such that the error reduction stagnates for shorter lags than without model errors. Overall, it should be desirable to be able to smooth over a long lag, because then more observations are taken into account. This will be particularly beneficial if the observations are incomplete.

Ensemble smoothers add the issue of sampling errors caused by small ensembles. Sampling errors in the initial ensemble matrix $\mathbf{X}_{0 \mid 0}^{a}$ will deteriorate the quality of the weight matrix $\mathbf{G}_{k}$ at all times of the assimilation process. This will lead to suboptimal filter analysis state estimates (through Eq. 18), but also suboptimal ensemble transformations in the smoother (Eq. 20). The additional error reduction from the filter error by the smoother will be smaller than without sampling errors. However, in a linear system, the smoother does still reduce the errors of the smoothed state estimates and converges for large lags.

Cosme et al. (2010) mention that the cross-correlation might fade with the time distance between filter analysis and smoothing due to model errors or nonlinearities. They demonstrate that for an erroneous parameterization of the model errors by a too small covariance inflation, the estimation errors can increase beyond a certain optimal lag. Their example used a nonlinear high-resolution ocean model, but it was not examined whether the error increase was due to the model error parameterization or the nonlinearity. While for a linear system, the crosscorrelations are ideally used for smoothing, the nonlinearity 
can result in cross-correlations that are inconsistent with the underlying linearity-assumption of the smoother. The optimality of the smoother does no longer hold due to these spurious cross-correlations. Thus, in case of nonlinearity, the smoother performance is influenced by a combination of existing, but fading cross-correlations as well as spurious cross-correlations. While the spurious cross-correlations result in a limitation of the smoother in reducing the estimation errors similar to the decorrelation, they can also deteriorate the smoothed state estimates for long lags. This combined effect will be examined in the following sections.

\section{Numerical Experiments}

\subsection{Experimental setup}

In this section, the behavior of the ESTKS is examined in identical twin experiments using the model by Lorenz (1996); Lorenz and Emanuel (1998), denoted below as L96 model. The L96 model is a simple nonlinear model that has been used in several studies to examine the behavior of different ensemble-based Kalman filters (e.g. Anderson 2001; Whitaker and Hamill 2002; Ott et al. 2004; Sakov and Oke 2008; Janjić et al. 2011). For the experiments, the implementation by Nerger et al. (2012a), including the regulated localization, has been extended by the smoother. The time integration is computed using the fourth-order Runge-Kutta scheme with a non-dimensional time-step size of 0.05 . The model as well as the filter and smoother algorithms are part of the Parallel Data Assimilation Framework (PDAF, Nerger et al. 2005; Nerger and Hiller 2013, http://pdaf.awi.de).

The model state dimension is set to $n=40$. At this dimension, the ESTKF can be successfully applied without localization. To examine the influence of the nonlinearity on the smoother without a possible inference by localization, results for global filters are discussed first. Subsequently, the influence of the localization is examined.

The nonlinearity of the L96 model can be controlled by a forcing parameter $F$ (see Lorenz and Emanuel 1998). The model results in a periodic wave with wave number 8 for $F \leq 4$. Perturbations of this wave are damped over time. For $F>4$, the model develops a non-periodic behavior. The strength of the nonlinearity can be specified by the Lyapunov time $\lambda^{-1}$, which is the asymptotic time at which a small deviation grows by a factor given by the exponential constant $e$. For the L96 model, $\lambda^{-1}$ was described by Karimi and Paul (2010) for $F>4$ by the function $\lambda^{-1}=$ $123.8 F^{-2.6}+0.158$. A related measure of error growth is the error doubling time (see, e.g., Lorenz and Emanuel 1998), which is approximately given by $\ln (2) \lambda^{-1}$.

For the twin experiments, a trajectory representing the truth is computed over 21000 time steps initialized with a state of constant value of 8.0 , but with $x_{20}=8.008$ (see Lorenz and Emanuel 1998). Observations of the full state are assimilated, which are generated by adding uncorrelated normally distributed random noise of variance one to the true trajectory. The observations are assimilated with an offset of 1000 time steps to omit the spin-up period of the model. The initial ensemble for all experiments is generated by second-order exact sampling from the variability of the true trajectory (see Pham 2001). All experiments are performed over 20000 time steps. The ensemble size is set to 34 members. The forgetting factor is tuned for each experiment to obtain a minimal estimation error.

To vary the nonlinearity of the experiments, two sets of experiments are conducted. First, the forcing parameter $F$ is varied between 1 and 10 to change the nonlinearity of the model dynamics. In these experiments the observations are assimilated after each time step. In the second set, the forcing parameter is kept constant and the time interval between the analysis steps is increased from 1 up to 9. With the longer forecast phases, the nonlinearity of the assimilation problem increases because the model nonlinearity acts longer on the ensemble states.

With the L96 model, the filter performance can depend on the initial ensemble (see, e.g., Fig. 4 of Nerger et al. 2012a). The experiments performed here are generally in the parameter regime where the filter estimates are robust. 
Thus, the estimation errors of the filter show only small variations in the time-mean estimation errors. However, the variation for different initial ensembles concerns the determination of the optimal smoother lag as will be visible in the error bars of the figures discussed below. To take this behavior into account, each experiment defined by the parameter set of forgetting factor, forcing parameter $F$, and length of forecast phase is repeated 10 times with different random numbers for the initial ensemble generation. The assimilation performance is then assessed using the analysis root mean square (RMS) error for each experiment over the last 18000 time steps of each experiment. The first 2000 time steps are omitted to exclude the initial transient phase of the assimilation from the computation. The RMS errors are then averaged over each set of 10 experiments with different random numbers for the ensemble generation. We refer to this mean error as MRMSE. The error bars for the optimal lag show the median, maximum, and minimum values for each set of 10 experiments. Because the filter itself is influenced by the nonlinearity, the MRMSE obtained with the smoother is considered relative to that of the filter.

\subsection{Smoother behavior with varying forcing}

Figure 1 shows the MRMSE as a function of the smoother lag for five different values of the forcing parameter $F$. The MRMSE is very small for $F \leq 4$ where the L96model exhibits a periodic behavior. For $F>4$, the MRMSE increases strongly. In addition, the typical influence of the smoother becomes visible. The MRMSE obtained with the filter is reduced by the smoother. For short lags, each additional lag results in a strong reduction of the MRMSE. However, for increasing lags the MRMSE curve flattens and reaches an asymptotic value where the MRMSE shows only a small variation for different lags. The common interpretation for the smoother performance as a function of the lag (see Khare et al. 2008; Cosme et al. 2010) is that for short lags, the cross-correlations between the ensembles at different times provide useful information to reduce the error in the state estimate. The cross-correlations fade over time due to model errors, dissipative dynamics, or nonlinearities of the dynamics. This results in a lower bound of the estimation errors. However, for the cases with $F>5$ a small increase of the MRMSE is observed at the beginning of the asymptotic regime (it is too small to be visible in Fig. 1). The increase shows that there is an influence of spurious correlations that can slightly deteriorate the smoothed state estimate.

As an effect of the nonlinearity, it is visible in Fig. 1 that the lag at which the asymptotic MRMSE is reached decreases for larger $F$. To quantify the influence of the forcing parameter on the smoother, the optimal lag $l_{\text {opt }}$ is considered. In general, $l_{\text {opt }}$ should be the lag at which the minimal MRMSE is obtained. However, as this happens in the range of lags where the MRMSE curve is very flat, we found more accurate results when $l_{o p t}$ is defined as the lag where the slope of the MRMSE as a function of the lag decreases below a limit. For the analysis below, the limit was set to $5 \cdot 10^{-6}$. This value was chosen to be as small as possible while avoiding fluctuation effects in measuring $l_{o p t}$ that would lead to overly big error bars.

The results shown in Fig. 1 are obtained with the choice of the forgetting factor for each forcing that results in the minimal MRMSE for the filter analysis. This optimal forgetting factor is $\rho=1.0$ for $F \leq 4.5$ and then decreases about linearly to $\rho=0.96$ for $F=10$. In the experiments discussed below, the same value of the forgetting factor was optimal for the filter and the smoother for all cases with $l_{\text {opt }}>0$. Thus, the forgetting factor does not need to be retuned if a smoother is added to a filtering system. If the forgetting factor is reduced below its optimal value, i.e. the inflation of the forecast covariances is too large, the value of the MRMSE increases, both for the filter and the smoother. In addition, $l_{o p t}$ is reduced. This is caused by larger changes of the state by the smoother due to the increased inflation. These erroneous changes impact the smoothing already for smaller lags and lead to a stronger increase of the MRMSE. If the inflation is reduced by increasing the forgetting factor 
from its optimal value, the filter tends to diverge. This effect is caused by the fact that the optimal inflation is close to the choice where filter divergence occurs (see Sakov and Oke 2008). Usually, the forgetting factor that results in the minimal MRMSE also resulted in the largest $l_{\text {opt }}$. If the forgetting factor would be kept fixed at a low value like 0.96, which is optimal for $F=10$, the inflation would be too big for weaker model forcing. In these cases, the MRMSE is larger than for the optimal choice of $\rho$ and $l_{o p t}$ is reduced. However, also with constant $\rho, l_{\text {opt }}$ decreases when the forcing is increased.

The solid line in the left panel of Fig. 2 shows $l_{o p t}$ for $m=34$ and different values of the forcing. The optimal lag is zero for the periodic cases $(F \leq 4)$. Thus, the filtered state estimate shows the smallest errors, while the smoothing increases the MRMSE. To interpret this behavior one has to consider that the MRMSE is computed over the last 18000 time steps of each experiment. As perturbations of the wave are damped by the model for $F \leq 4$, there is the special situation that the filter can already yield the best state estimate. Mainly, the data assimilation speeds up the convergence to the truth during the initial transient phase. At the beginning of the assimilation process, the smoothing is also beneficial and $l_{\text {opt }}$ is larger than zero. However, after the transient phase, the smoothing results in an over-fitting to the data, which has much larger errors with a standard deviation of one. Hence, the smoother increases the errors.

In the non-periodic cases $(F>4)$, perturbations of the wave are amplified by the model dynamics. For $F=4.5$, $l_{\text {opt }}$ jumps to the largest tested lag of 200 time steps, both for the MRMSE computed over 18000 time steps or the full 20000 time steps of each experiment. With growing forcing, $l_{\text {opt }}$ shrinks and reaches 58 time steps for $F=10$. Figure 2 shows also the estimated error doubling time multiplied by 7. Between $F=4.5$ and $8, l_{\text {opt }}$ is close to this function. However, for larger $F, l_{\text {opt }}$ decreases slower than the error doubling time.

The right panel of Fig. 2 shows the MRMSE at $l_{\text {opt }}$ for $m=34$ as a function of the forcing parameter. For
$F>4$, the MRMSE first increases strongly, but then the growth becomes slower. The same behavior is visible for the MRMSE obtained with the filter. Comparing the MRMSE for the filter with the minimum MRMSE obtained with the smoother, one finds that the smoother reduces the MRMSE to about $50 \%$ for all forcings with $F>4$.

The previous studies on ensemble smoothing (Khare et al. 2008; Cosme et al. 2010) stress the impact of sampling errors on the smoother performance. Further, the effect of sampling errors in increasing the need of the ensemble inflation was discussed by Bocquet (2011). The optimal lag will obviously be influenced not only by the nonlinearity of the dynamics but also by sampling errors. In the experiments, the standard deviation of the observation errors is kept constant, such that the sampling errors in the observations are constant on average. Further, the degrees of freedom for the analysis are constant for a fixed ensemble size. Finally, due to the used 2nd-order exact sampling, the relevant errors are well sampled as long as the number of positive Lyapunov exponents that result in dynamical instabilities is smaller than the ensemble size. This condition is fulfilled, as for $F=10$ the number of positive Lyapunov instabilities is 14 (Lorenz and Emanuel 1998) while it is less for smaller $F$. For $F=8$, the experiments with localization in section 2.2 also confirm that the sampling is very good for $m=34$. To examine the influence of sampling errors caused by a smaller ensemble, Fig. 2 shows also $l_{\text {opt }}$ for an ensemble of 20 states. The smaller ensemble results in a curve that is parallel to that for $m=34$ but shifted to smaller lags. For $F>4.5, l_{\text {opt }}$ for $m=20$ is about 4.5 times the error doubling time. In addition, the error reduction due to smoothing is only about $40 \%$. If also the observational information is further reduced by observing only each second grid point, the optimal lag is reduced to 4.0 times the error doubling time and the error reduction by the smoother is reduced to about 35\% (not shown).

Overall, the experiments with varying forcing show that the decorrelation of the ensembles at the smoother and 
filter times occurs over shorter time intervals for stronger nonlinearity. The optimal lag of the smoother is a few times the error doubling time of the model. The factor to the error doubling time depends on the sampling errors and shrinks for larger sampling errors. The relative error reduction by the smoother is decreased if the sampling errors are larger due to a smaller ensemble. However, the relative error reduction did not show a dependence on the model forcing.

\subsection{Smoother behavior with increasing forecast length}

Increasing the forecast length for a fixed forcing parameter $F$ also increases the nonlinearity of the data assimilation process. However, while the forcing determines the nonlinearity of the model dynamics, the forecast length $\Delta t$ determines the length over which the nonlinear dynamics act on the ensemble members.

To assess the influence of the forecast length, experiments with fixed forcing values of between 5 and 8 are performed. As in the case of varying forcing, the optimal choice of the forgetting factor depends on $\Delta t$. For $F=5$ it shrank about linearly from $\rho=0.99$ when assimilating at each time step to $\rho=0.85$ for $\Delta t=9$ time steps. A much stronger inflation was required for $F=8$ where $\rho$ had to be reduced to 0.51 for $\Delta t=9$ to obtain stable results with minimal errors. Because the ensemble size and observation error variance are kept constant, sampling errors are also constant in these experiments.

When the nonlinearity in the assimilation process is increased by larger choices of $\Delta t$, the influence of spurious correlations grows. The spurious correlations are caused by the violation of the assumption that the cross-correlations between the ensembles at the filter analysis time $k$ and the smoother time $k-\Delta t$ are linear (see section 2.4). The spurious correlations result in a smaller error-reduction by the smoother and in a stronger error increase when the lag is increased beyond $l_{\text {opt }}$. The largest error increase beyond $l_{o p t}$ was obtained for $\Delta t=9$, hence for the largest nonlinearity tested in the experiments. For $F=8$ and $\Delta t=9$, the smoother impact to decrease the RMS error was reduced to only $25 \%$ of its maximal value at $l_{\text {opt }}$. If the inflation was not carefully tuned, the smoother state estimate could even be worse than the estimate of the filter.

If $\Delta t$ is increased for a fixed value of $F, l_{\text {opt }}$ decreases as is shown in the left panel of Fig. 3. As before, a larger forcing results in a smaller $l_{\text {opt }}$. This holds for all forecast lengths. Compared to the case when observations are assimilated at each time step, a forecast length of 9 time steps resulted in a reduction of $l_{\text {opt }}$ to $51 \%$ for $F=5$ and to about $25 \%$ for $F=8$. In the latter case, $l_{\text {opt }}$ is only 18 time steps, which corresponds to $2 \Delta t$ or about twice the error doubling time.

The increase in nonlinearity caused by larger $\Delta t$, also reduces the performance of the smoother relative to the filter. The right panel of Fig. 3 illustrates the influence by showing the MRMSE for the smoother at $l_{\text {opt }}$ and the MRMSE for the filter. The MRMSE grows for increasing forcing. For fixed forcing, the MRMSE of both the filter and the smoother grow about linearly with $\Delta t$. The increase is faster for larger forcing. For the larger forcing, the impact of the smoother on the MRMSE for the assimilation with $\Delta t=1$ increases (see also Fig. 2). However, the ability of the smoother to reduce the estimation errors with growing forecast length decreases for larger forcing. For $F=5$, the difference between the MRMSE for the filter and the smoother is almost constant. However, the difference between the MRMSE for the filter and the smoother decreases for growing $\Delta t$ in case of the larger forcings. In the case of the largest nonlinearity $(F=8$, $\Delta t=9$ the error reduction is only $0.7 \%$.

The experiments show that the different influences of the forcing on the model nonlinearity and the forecast length on the duration of the nonlinear action of the dynamics during the ensemble integration result in different influences on the smoother performance. The stronger nonlinearity reduces $l_{\text {opt }}$ in both cases. This is caused by a combination of a faster decorrelation of the ensembles at the filter and smoother times and by spurious correlations that violate the assumption of linear correlations in the smoother. 
However, while for short forecast lengths the stronger model nonlinearity results in a larger positive influence of the smoother, this influence is strongly reduced for long forecasts.

\subsection{Influence of localization}

The experiments discussed in the previous sections used the global filter to assess the impact of the nonlinearity on the smoother performance without a possible influence of localization. However, high-dimensional data assimilation applications typically require the use of localization. The localization down-weights or even removes long-range covariances between different grid points. This method should also have an effect on the smoother, which is studied in this section. Here, the ESTKF algorithm with observation localization is applied with the L96 model with a fixed forcing parameter of $F=8$. Ensemble sizes $m=34$ and $m=20$ are used to assess the impact of the localization in cases where the global filter is also successful. However, the localization allows to use even smaller ensembles. To examine the impact of small ensembles, $m=15$ and $m=10$ are also considered below. As in the previous experiments, the forgetting factor $\rho$ was tuned to obtain minimal RMS errors. Values between 0.98 and 0.9 were required. The forgetting factor was closest to one for those localization lengths where minimal RMS errors were obtained.

Localization in smoothers with the L96 model was also discussed by Khare et al. (2008). That study used a different sampling method from our experiments. However, the results discussed here are consistent with the previous study but provide more insights into the effect of localization on smoothing.

For the localized filter and smoother, the top left panel of Fig. 4 shows the MRMSE as a function of the lag for the four different ensemble sizes. For each ensemble size, the localization radius was chosen so that the MRMSE of the filter was minimal. As expected, the MRMSE grows with decreasing ensemble size. Also it is visible that the optimal lag shrinks when the ensemble size is reduced.

The optimal lag $l_{\text {opt }}$ as a function of the localization radius is shown in the top right panel of Fig. 4. Localization radii up to 80 grid points have been used for the two larger ensembles. With the smaller ensembles, the assimilation becomes unstable with very large error bars for larger localization radii. The figure focusses on the parameter region where stable results are obtained. As the model domain has a size of 40 grid points, a localization radius of more than 20 grid points implies that all observations are used for each local analysis. However, the weight of the observations is still reduced according to their distance. The localization radii that result in the biggest $l_{\text {opt }}$ are rather large with 50 grid points for $m=20$ and 80 grid points for $m=34$. The optimal lags are longer with localization than for the global analysis. Thus, the smoother profits from the reduction of the influence of spatial long-range covariances by localization.

The influence of the localization is also visible in the MRMSE shown in the bottom left panel of Fig. 4. The MRMSE for both the filter and the smoother are smaller with localization than for the global analysis. For $m=34$, the improvement is very small with $1.5 \%$ for the filter and $5.6 \%$ for the smoother, which shows that the sampling quality of the ensemble is very high. However, even for $m=$ 34 , the filter and the smoother profit from the localization. For $m=20$, the localization shows a bigger influence due to the larger sampling errors in the ensemble-estimated state error covariance matrix. With localization the MRMSE of the smoother is reduced by up to $32 \%$ compared to the global analysis. These results are qualitatively consistent with those reported by Khare et al. (2008). However, the experiments also show that the positive influence of the localization is larger for the smoother than for the filter. This effect is visible in the bottom right panel of Fig. 4 that shows the difference in the MRMSE from the smoother and the filter. For $m=34$ and $m=20$, the error reduction by the smoother is larger with localization than with the global 
analysis. Also, there is an optimal localization radius where the relative impact of the smoother is the largest.

Small ensembles can only be used with localization. The required localization radius decreases with the ensemble size. For $m=15$ and $m=10$, Fig. 4 shows that also $l_{\text {opt }}$ decreases with the ensemble size. In addition, the MRMSE of the filter and the smoother increase. However, the bottom left panel of Fig. 4 shows that the smoother still has a large impact on the MRMSE even for $m=10$. The localization radius significantly influences the MRMSE of the smoother and the filter. The smallest MRMSE of the filter as well as the smoother are obtained for the same localization radius. The bottom right panel of Fig. 4 also shows that the differences between the MRMSE obtained with the filter and smoother become smaller if the ensemble size is reduced. Thus the positive effect of the smoother is reduced. This is in accordance with the inferior sampling quality of the ensemble covariance matrix for decreasing ensemble sizes. The sampling errors deteriorate the weight matrix $\mathbf{G}_{k}$, which results in the overall larger MRMSE. The smoother is more strongly influenced by the sampling error than the filter, because the weight matrices $\mathbf{G}_{i}$ are reused to perform the smoothing. The inferior sampling quality also leads to the reduced $l_{\text {opt }}$ for smaller ensembles. Conversely, the fact that the difference between the MRMSE of the filter and the smoother (Fig. 4, bottom right) grows for larger ensembles indicates that the smoother profits more from the improved sampling than the filter.

For all ensemble sizes, Fig. 4 shows that the MRMSE for the smoother and the filter are smallest for the same localization radius. In addition, the error reduction by the smoother from the MRMSE of the filter (bottom right panel of Fig. 4) is maximal for this localization radius. Thus, the effect of minimizing the MRMSE of the filter by varying the localization radius is amplified in the smoother.

Overall, the experiments show that the influence of localization on the smoother is analogous to its influence on the filter. The same localization radius results in minimal MRMSE for both the filter and the smoother. The
MRMSE from the smoother is slightly more reduced by the localization than the filter errors. The optimal lag also varies with the localization radius and becomes maximal for a localization radius slightly bigger than the value where the errors are minimal.

\section{Smoother behavior with a global ocean model}

\subsection{Experimental setup}

The experiments with the small L96 model allow to vary the nonlinearity in a systemic way. However, the model includes only simplified dynamics. To obtain insight in the smoother behavior with a complex model, twin data assimilation experiments with the finite-element sea-ice ocean model (FESOM, Danilov et al. 2004; Wang et al. 2008; Timmermann et al. 2009) are conducted. FESOM uses finite elements to solve the hydrostatic ocean primitive equations. Unstructured triangular meshes are used, which allow for a varying resolution of the mesh. A global configuration with a horizontal resolution of about $1.3^{\circ}$ and refinement in the equatorial region (see Wang et al. 2012) is used here. The model uses 40 vertical levels. The model is forced by the interannually varying data documented by Large and Yeager (2009). The global ocean model configuration shows only weak nonlinearity, which could only be increased by generating a higher-resolved model mesh. Accordingly, the experiments can only access the behavior of the smoother in case of the complex model without examining its dependence on nonlinearity.

For the data assimilation, FESOM was coupled to PDAF (Nerger et al. 2005; Nerger and Hiller 2013) into a single program that allows to perform the assimilation experiments on parallel computers. The state vector has a size of about 10 million. It includes the sea surface height (SSH) and the 3-dimensional fields temperature, salinity, and the velocity components. For the twin experiments, the model was initialized from a spin-up run and a trajectory over one year was computed. This trajectory contains the model fields at each tenth day and represents the "truth" for 
the assimilation experiments. An ensemble of 32 members is used, which is generated by second-order exact sampling from the variability of the true trajectory (see Pham 2001). The initial state estimate is given by the mean of the true trajectory. Pseudo observations of the SSH at each surface grid point are generated by adding uncorrelated random Gaussian noise with a standard deviation of $5 \mathrm{~cm}$ to the true model state. The analysis step is computed after each forecast phase of 10 days with an observation vector containing about 68000 observations. Each experiment was conducted over a period of 360 days.

The experiments use the ESTKF with observation localization. The localization length was set to $1000 \mathrm{~km}$. This choice resulted in the smallest time-mean RMS errors compared to other radii in the the range between 600 and $1400 \mathrm{~km}$. A forgetting factor $\rho=0.9$ is used to inflate the estimated variance of the forecast ensembles.

\subsection{Impact of smoothing with the global ocean model}

The impact of assimilating the pseudo observations is assessed in terms of the RMS error for each analysis time. Figure 5 shows the RMS error over time. As the mean state is a rather unrealistic initial state estimate, the RMS error is already strongly reduced at the first analysis time. After about 5 analysis cycles, the assimilation process reaches its asymptotic phase during which the RMS error shows only small variations. The RMS error for the smoother is displayed in Figure 5 for a lag of 17 analysis steps, corresponding to 170 days, which is the optimal lag computed as in section 3 .

The time-mean RMS error obtained with the smoother for varying lags relative to the error obtained with the filter is shown in the left panel of Fig. 6. The error is averaged over the asymptotic period (days 60 to 360) of the experiment. The smoother reduces the RMS error by up to $9 \%$. Small lags up to about 100 days have the strongest effect in reducing the RMS error from its value without smoothing, but up to about 200 days, the further error reduction is still visible.

Copyright (c) 0000 Royal Meteorological Society
Overall, the effect of the smoother on the sea surface height is very similar to the case of the small L96 model. For shorter lags, each additional previous analysis step that is smoothed has a strong positive effect. The effect of the smoothing then stagnates such that for longer lag lengths the RMS error remains almost constant. For the complex ocean model, it is visible that smoother lags up to 200 days have positive impacts, with each additional lag being beneficial in reducing the RMS error. The strongest impact is achieved by the smoothing over the first lags up to 50 days. This behaviour of the smoother is similar to that described by Cosme et al. (2010). However, that study found shorter optimal lags, which is likely due to the finer resolution of their model and more frequent analysis steps.

With respect to computing times, the extension of the filter method by the smoothing adds only a little time. For the FESOM model, the ensemble integration was fully parallelized, thus 32 model integrations were performed concurrently. A single experiment took about 5300 seconds using 2048 processor cores. The analysis step was computed using 64 processor cores. For the filter alone, the analyses took about 50 seconds. The smoothing took up to 100 seconds for the longest lag of 35 analysis steps. Thus, the filter analysis and smoothing took less than $3 \%$ of the execution time. Nonetheless, the time for smoothing can exceed the time required for the application of the filter for long lags. Apart from the computing time, the memory requirement can become limiting for long lags. This is because the past state ensemble needs to be stored for each smoothed lag.

\subsection{Multivariate impact of smoothing}

The assimilation experiments with the FESOM model perform multivariate assimilation. Thus, next to the observed SSH fields, all other fields in the state vector are updated by the filter and the smoother. The multivariate corrections utilize the ensemble-estimated cross covariances between the observed SSH field and the unobserved fields.

Q. J. R. Meteorol. Soc. 00: 1-?? (0000) 
The right panel of Fig. 6 shows the relative RMS error averaged over the asymptotic period (days 60 to 360) for the 3-dimensional temperature and salinity fields as well as the two horizontal velocity components. The relative RMS errors for the different fields depend differently on the lag. For salinity, the error decreases by up to $2.9 \%$ for long lags similarly to the decrease in the error of the sea surface height. For the temperature and the zonal velocity fields, the relative RMS errors decrease up to a lag of 40 days and increase again for longer lags. For the meridional velocity a strong decrease of the error is visible up to a lag of 30 days. For lags between 40 and 170 days, the error shows a further slow decrease. The error decrease then stagnates, but for lags beyond 250 days the error increases slighty.

The optimal lags for the different fields are summarized in Table I together with the error reduction obtained by the smoother. The optimal lags are distinct for the different fields. While the optimal lags for temperature and zonal velocity are relatively short with 40 days, the optimal lags for the other fields are quite long with 170 to 180 days. A short optimal lag for the temperature field was attributed by Brusdal et al. (2003) to the direct influence of the surface temperature to the surface temperature forcing applied to the model.

The different optimal lags point to the question how to define the optimal lag in multivariate smoothing. When the short lag of 40 days would be used, which is optimal for temperature and zonal velocity, the observational information is not optimally used for the other model fields. On the other hand, a long lag of 170 days would be far beyond the optimal lag for temperature and zonal velocity. At the ocean surface this long lag would even deteriorate the meridional velocity field (not shown). Instead of using a cautiously chosen short lag, one might consider to perform the smoothing over distinct lags for the different fields. This method could optimize the impact of the smoother for each field, but it could also negatively affect the balances in the model state.

Copyright (c) 0000 Royal Meteorological Society
Considering that the localization radius was set to $1000 \mathrm{~km}$ one might worry whether the long lags of up to 180 days are physically plausible. The expectation is that the circulation information should have moved out of a localization region within a few weeks. The experiments show that most of the observational information is used for lags up to 50 days. For longer lags the changes to the model fields caused by the smoother are about one order of magnitude smaller than for short lags. However, the changes to the model fields are similarly distributed over the whole model domain for both the short and long lags. The smoother does not improve the state estimate at all locations, however, for short lags the improvements dominate clearly. Over all, the experiments do not allow to conclude whether the improvements for long lags are an effect of the numerics or whether not all information at all places in the global ocean mesh has been advected away allowing for further successful smoothing.

The multivariate assimilation utilizes the estimated cross-covariances between the $\mathrm{SSH}$ field and the other fields. A larger ensemble is expected to better represent the cross-covariances. The experiments with the L96 and localization indicated that an increasing ensemble size can improve the smoother to a larger amount than the filter. Increasing the ensemble size for the assimilation with FESOM from 32 to 48 members does also improve the estimates by both the filter and the smoother. However, the smoother impact grows with the increasing ensemble. The change in the error reduction varies for the different fields. It is smallest for the $\mathrm{SSH}$ with about $4 \%$ and largest for the meridional velocity with $43 \%$ for a lag of 170 days. Thus, also in case of the complex global ocean model, the smoother does profit more from increasing the ensemble size than the filter.

\section{Summary and Conclusion}

This study examined how an ensemble smoother is affected by the nonlinearity in a numerical model and by localization. A smoother based on the error-subspace Q. J. R. Meteorol. Soc. 00: 1-?? (0000) 
transform Kalman filter (ESTKF) was used in the numerical experiments. However, analogous results can be expected with smoother extensions of filters like the ETKF and the SEEK filter, as the equations of these algorithms are very similar to those of the ESTKF.

Twin experiments with the 40-dimensional Lorenz96 model showed an optimal smoothing lag at which the root mean square error of the state estimate was minimal. The experiments demonstrated that, with a nonlinear model, the smoother can utilize the observational information to reduce the estimation errors for lags up to a few times the error doubling time of the model. Thus, the optimal lag becomes shorter when the nonlinearity of the model dynamics increases. The optimal lag was between 2 and 8 times the error doubling time of the Lorenz96 model, but depended also on the ensemble size that influenced the sampling errors in the experiments. For a fixed ensemble size, the factor of the optimal lag to the error doubling time was nearly constant if the model nonlinearity was varied. The optimal lag also decreased with increasing nonlinearity of the assimilation process caused by longer forecast phases. The experiments with the Lorenz96 model also showed that the overall error-reduction that can be obtained with the smoother depends on the nonlinearity of the model dynamics that was controlled by a forcing parameter. If the time interval between successive analysis steps was increased, the maximal reduction of the RMS errors by the smoother remained almost constant for small nonlinearity where the forecast length was well below the error doubling time. For larger forcing, when the maximal forecast length was closer to the error doubling time, the influence of the smoother decreased with growing forecast lengths.

The application of localization resulted in a stronger improvement of the smoothed state estimates compared to the filter estimates without smoothing. Thus, the smoother profits more than the filter from the damping of spurious correlations by the localization. The performance of the smoother to reduce the errors of the filter depended on the localization radius. However, the smoother showed the strongest reduction of the errors from the filter for the same localization radius for which the filter provided the smallest errors. The optimal lag decreased with the ensemble size due to the reduced sampling quality of the ensemble covariance matrix.

The experiments in this study used an inflation of the ensemble covariance matrix with a constant forgetting factor that was tuned to obtain the smallest possible RMS errors for the filter. The tuning showed that the filter and the smoother result in minimal RMS errors for the same choice of the forgetting factor. Thus, the tuning performed for a filter with constant forgetting factor is likewise valid for the smoother. Accordingly, the smoother can be added to an existing assimilation system without re-tuning the filtering system. However, the smoother lag should be carefully chosen to maximize the benefit of the smoother.

Experiments using a global ocean circulation model with weak nonlinearity showed results that are consistent with those obtained with the Lorenz96 model. Also here the smoother can be applied using the same inflation as tuned for the filter. The study of the multivariate smoother impact showed distinct optimal lags for different fields. Thus, one either needs to choose the same lag for all fields as a compromise and one might choose different lags for different model fields.

Synthetically generated observations were used in the experiments performed here. In case of real observations, possible representativeness errors of the observations as well as biases will also influence the smoother. This influence will be considered in a future study.

Increasing the ensemble size had a stronger positive effect on the smoothed than on the filtered fields for both models. Thus, as the ensemble size is typically chosen upon the consideration of computing cost and quality of the state estimates from the assimilation process, it might be worth reconsidering the ensemble size when a smoother is added to a filtering system. 


\section{Acknowledgement}

The authors are grateful to the Editor, Dr. M. Bocquet, as well as three anonymous reviewers for their comments that helped to improve the text. This work was partly funded by the SANGOMA EU project (grant FP7-SPACE-2011-1-CT283580-SANGOMA).

\section{References}

Anderson JL. 2001. An Ensemble Adjustment Kalman Filter for data assimilation. Mon. Wea. Rev. 129: 2884-2903.

Bishop CH, Etherton BJ, Majumdar SJ. 2001. Adaptive sampling with the Ensemble Transform Kalman Filter. Part I: Theoretical aspects. Mon. Wea. Rev. 129: 420-436.

Bocquet M. 2011. Ensemble Kalman filtering without the intrinsic need for inflation. Nonl. Proc. Geoph. 18: 735-750.

Brusdal K, Brankart JM, Halberstadt G, Evensen G, Brasseur P, van Leeuwen PJ, Dombrowsky E, Verron J. 2003. A demonstration of ensemble based assimilation methods with a layered OGCM from the perspective of operational ocean forecasting systems. J. Mar. Syst. 4041: $253-289$.

Cohn SE, Sivakumaran NS, Todling R. 1994. A fixed-lag Kalman smoother for retrospective data assimilation. Mon. Wea. Rev. 122: 2838-2867.

Cosme E, Brankart JM, Verron J, Brasseur P, Krysta M. 2010. Implementation of a reduced rank square-root smoother for high resolution ocean data assimilation. Oce. Mod. 33: 87-100.

Cosme E, Verron J, Brasseur P, Blum J, Auroux D. 2012. Smoothing problems in a Bayesian framework and their linear Gaussian solutions. Mon. Wea. Rev. 140: 683-695.

Danilov S, Kivman G, Schröter J. 2004. A finite-element ocean model: Principles and evaluation. Ocean Modeling 6: 125-150.

Evensen G. 2003. The Ensemble Kalman Filter: Theoretical formulation and practical implementation. Ocean Dynamics 53: 343-367.

Evensen G. 2004. Sampling strategies and square root analysis schemes for the EnKF. Ocean Dynamics 54: 539-560.

Evensen G, van Leeuwen PJ. 2000. An ensemble Kalman smoother for nonlinear dynamics. Mon. Wea. Rev. 128: 1852-1867.

Fisher M, Leutbecher M, Kelly GA. 2005. On the equivalence between kalman smoothing and weak-constraint four-dimensional variational data assimilation. Q. J. Roy. Meteor. Soc. 131: 3235-3246.

Gaspari G, Cohn SE. 1999. Construction of correlation functions in two and three dimensions. Q. J. Roy. Meteor. Soc. 125: 723-757.
Hunt BR, Kostelich EJ, Szunyogh I. 2007. Efficient data assimilation for spatiotemporal chaos: A local ensemble transform Kalman filter. Physica D 230: 112-126.

Janjić T, Nerger L, Albertella A, Schröter J, Skachko S. 2011. On domain localization in ensemble based Kalman filter algorithms. Mon. Wea. Rev. 139: 2046-2060.

Karimi A, Paul MR. 2010. Extensive chaos in the lorenz-96 model. Chaos 20: 043105.

Khare SP, Anderson JL, Hoar TJ, Nychka D. 2008. An investigation into the application of an ensemble kalman smoother to high-dimensional geophysical systems. Tellus 60A: 97-112.

Large W, Yeager S. 2009. The global climatology of an interannually varying air-sea flux data set. Clim. Dyn. 33: 341-364.

Lorenz EN. 1996. Predictability - a problem partly solved. In: Proceedings Seminar on Predictability. ECMWF, Reading, UK, pp. $1-18$.

Lorenz EN, Emanuel KA. 1998. Optimal sites for supplementary weather observations: Simulation with a small model. J. Atm. Sci. 55: $399-414$.

Nerger L, Danilov S, Hiller W, Schröter J. 2006. Using sea level data to constrain a finite-element primitive-equation ocean model with a local SEIK filter. Ocean Dynamics 56: 634-649.

Nerger L, Hiller W. 2013. Software for ensemble-based data assimilation systems - implementation strategies and scalability. Computers \& Geosciences 55: 110-118.

Nerger L, Hiller W, Schröter J. 2005. PDAF - the Parallel Data Assimilation Framework: Experiences with Kalman filtering. In: Use of High Performance Computing in Meteorology - Proceedings of the 11. ECMWF Workshop, Zwieflhofer W, Mozdzynski G (eds). World Scientific, pp. 63-83.

Nerger L, Janjić T, Schröter J, Hiller W. 2012a. A regulated localization scheme for ensemble-based Kalman filters. Q. J. Roy. Meteor. Soc. 138: $802-812$.

Nerger L, Janjić T, Schröter J, Hiller W. 2012b. A unification of ensemble square root Kalman filters. Mon. Wea. Rev. 140: 2335-2345.

Ott E, Hunt B, Szunyogh I, Zimin AV, Kostelich EJ, Corazza M, Kalnay E, Patil DJ, Yorke JA. 2004. A local ensemble Kalman filter for atmospheric data asimilation. Tellus 56A: 415-428.

Pham DT. 2001. Stochastic methods for sequential data assimilation in strongly nonlinear systems. Mon. Wea. Rev. 129: 1194-1207.

Ravela S, McLaughlin D. 2007. Fast ensemble smoothing. Oce. Dyn. 57: 123 .

Sakov P, Oke PR. 2008. Implications of the form of the ensemble transformation in the ensemble square root filters. Mon. Wea. Rev. 136: $1042-1053$. 
Timmermann R, Danilov S, Schröter J, Böning C, Sidorenko D, Rollenhagen K. 2009. Ocean circulation and sea ice distribution in a finie element global sea ice-ocean model. Oce. Mod. 27: 114-129.

van Leeuwen PJ. 2001. An ensemble smoother with error estimates. Mon. Wea. Rev. 129: 709-728.

van Leeuwen PJ, Evensen G. 1996. Data assimilation and inverse methods in terms of a probabilistic formulation. Mon. Wea. Rev. 124: 2898-2913.

Wang Q, Danilov S, Schröter J. 2008. Finite element ocean circulation model based on triangular prismatic elements with application in studying the effect of topography representation. J. Geophys. Res. 113: $\mathrm{C} 05015$.

Wang X, Wang Q, Sidorenko D, Danilov S, Schröter J, Jung T. 2012. Long-term cean simulations in FESOM: evaluation and application in studying the impact of Greenland Ice Sheet melting. Oce. Syn. 62: 1471-1486.

Whitaker JS, Hamill TM. 2002. Ensemble data assimilation without perturbed observations. Mon. Wea. Rev. 130: 1913-1927. 
Table I. Impact of the smoother relative to the filter analysis for the mean RMS error over 360 days.

\begin{tabular}{lcc}
\hline \hline Field & error reduction & optimal lag \\
\hline SSH & $9.0 \%$ & 170 \\
temperature & $1.0 \%$ & 40 \\
salinity & $2.9 \%$ & 180 \\
merid. velocity & $1.3 \%$ & 170 \\
zonal velocity & $0.9 \%$ & 40 \\
\hline
\end{tabular}

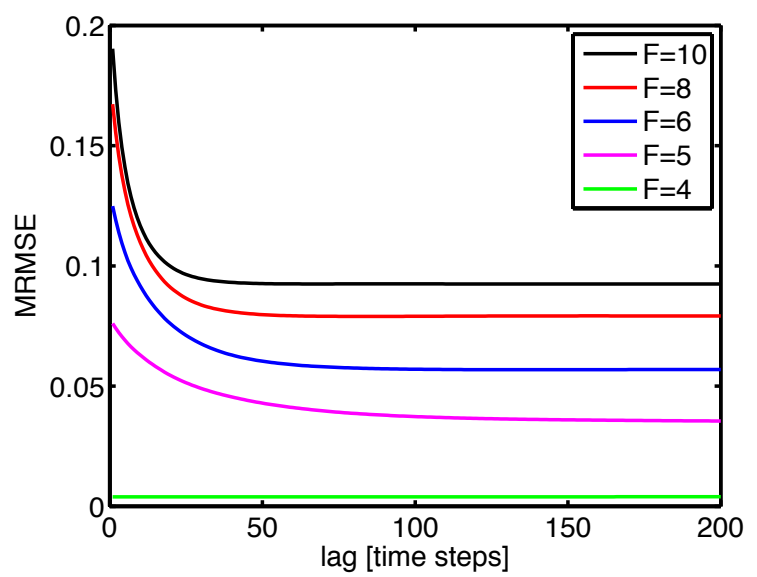

Figure 1. Mean RMS error (MRMSE) in dependence on the smoother lag for five cases with different forcing $F$.
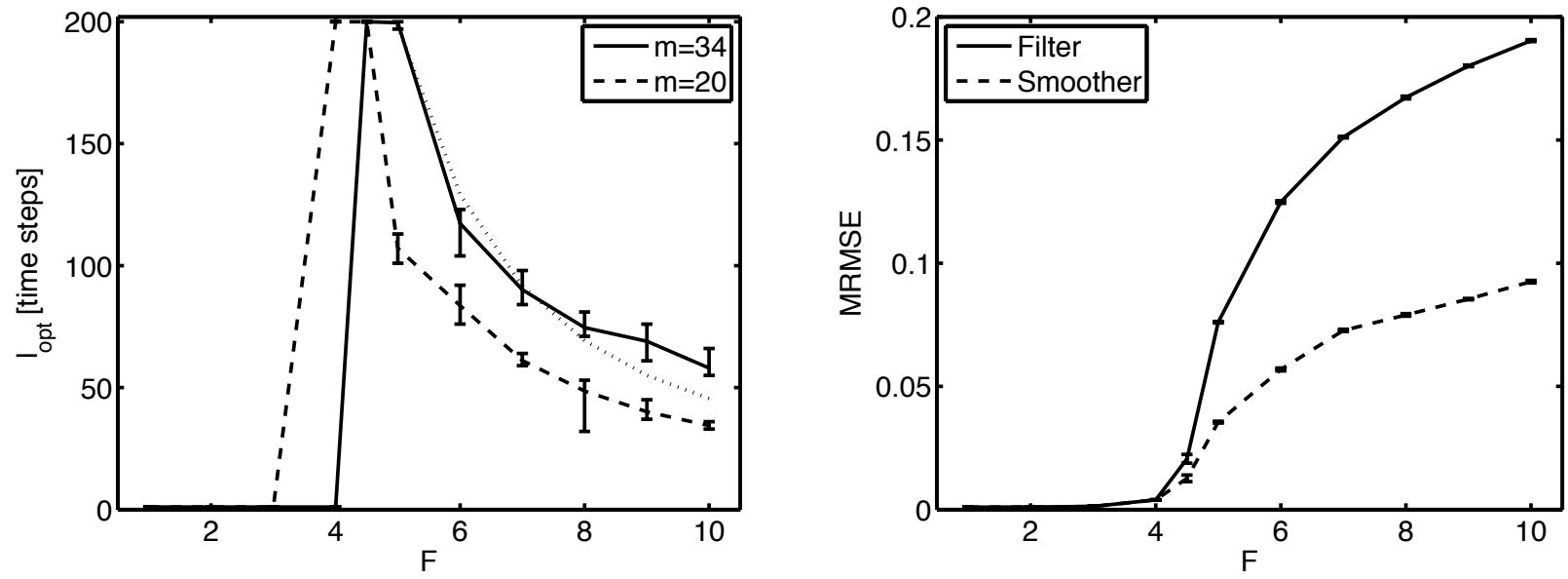

Figure 2. Left: Optimal smoother lag $l_{\text {opt }}$ as a function of the forcing $F$ for $m=34$ (solid) and $m=20$ (dashed). The estimated error doubling time multiplied by 7 (dotted) is very similar to the optimal lag for $m=34$. Right: Minimum MRMSE for $m=34$ at the optimal lag in dependence of the forcing $F$ (dashed) and MRMSE for the filter (solid). 

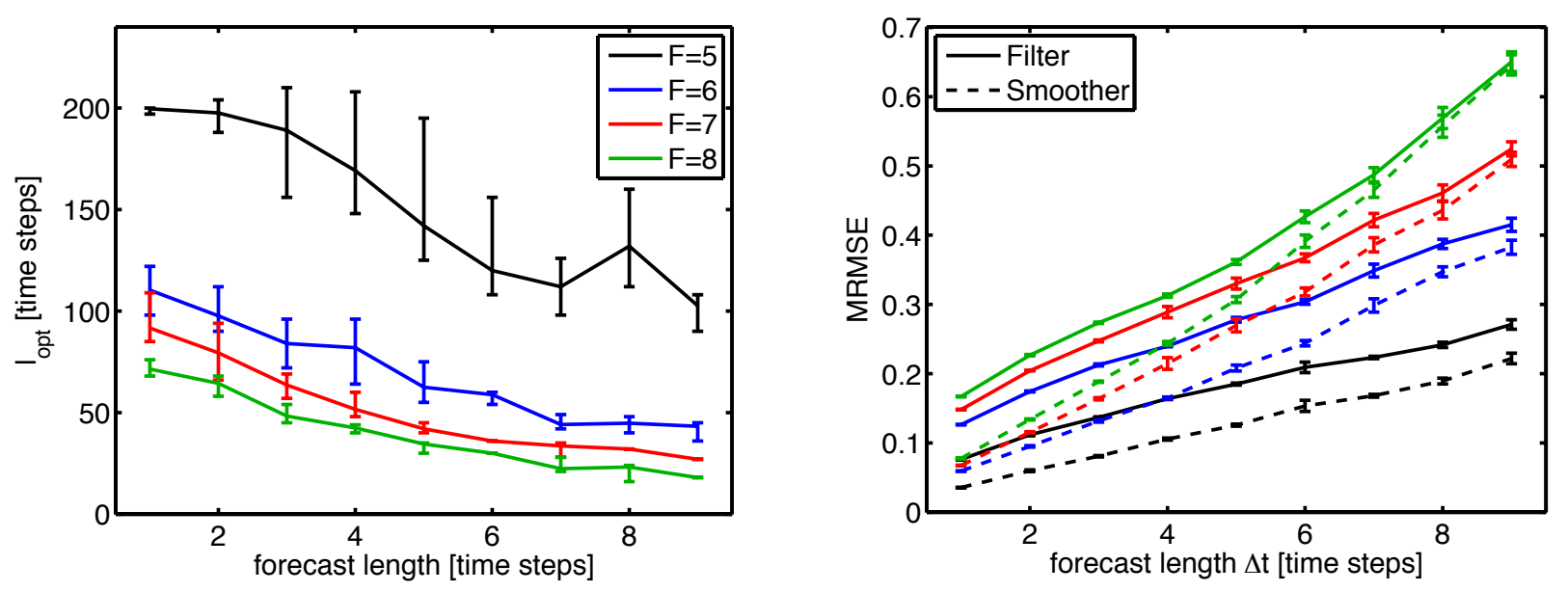

Figure 3. Left: Optimal smoother lag $l_{\text {opt }}$ as a function of the observation interval for four different forcings. The error bars show the variation of the optimal length over the 10 repetitions of each experiment. Right: Minimum MRMSE at $l_{\text {opt }}$ in dependence of the forecast length (dashed line). Shown is also the MRMSE for the filter (solid line).
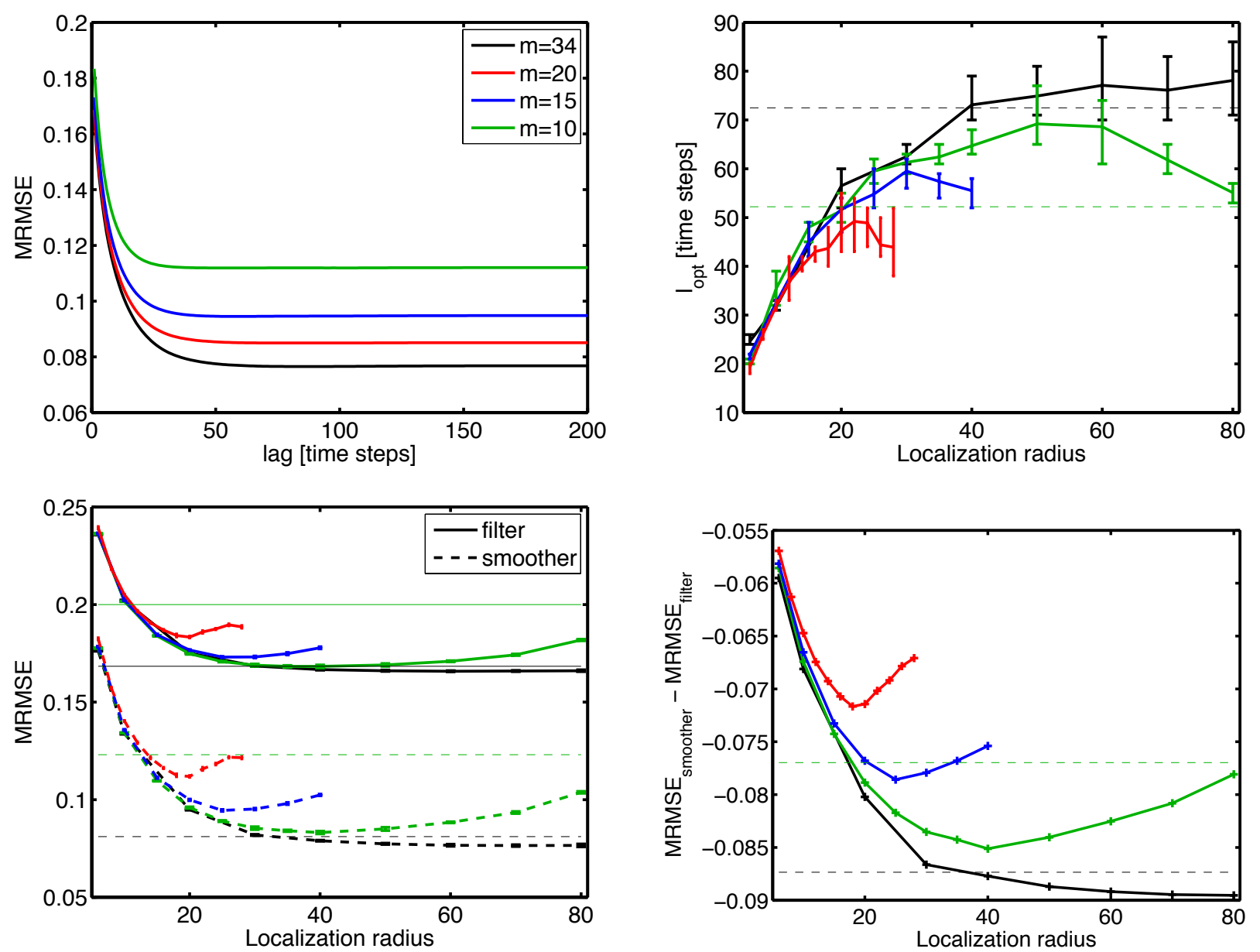

Figure 4. Behavior of the smoothing in case of localization. Top left: MRMSE as a function of the lag for the optimal localiztion radius, Top right: $l_{\text {opt }}$ as a function of the localization length. Bottom left: Minimum MRMSE at $l_{\text {opt }}$ in dependence of the localization length (dashed) and MRMSE for the filter (solid). Bottom right: Difference of the MRMSE of the smoother and the filter. The thin dashed lines show the values for the global analysis for $m=34$ and $m=20$. For the smaller ensembles a global analysis is not possible. 


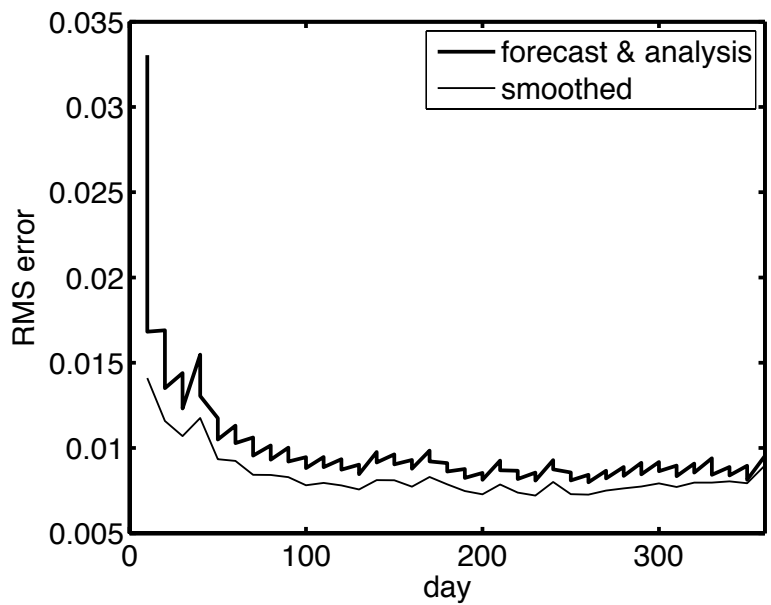

Figure 5. RMS errors over time for the assimilation of pseudo SSH data into the global FESOM model. (thick line) Saw tooth line showing RMS errors from alternating forecasts and analysis steps; (thin line) the smoothed state for a lag of 170 days.
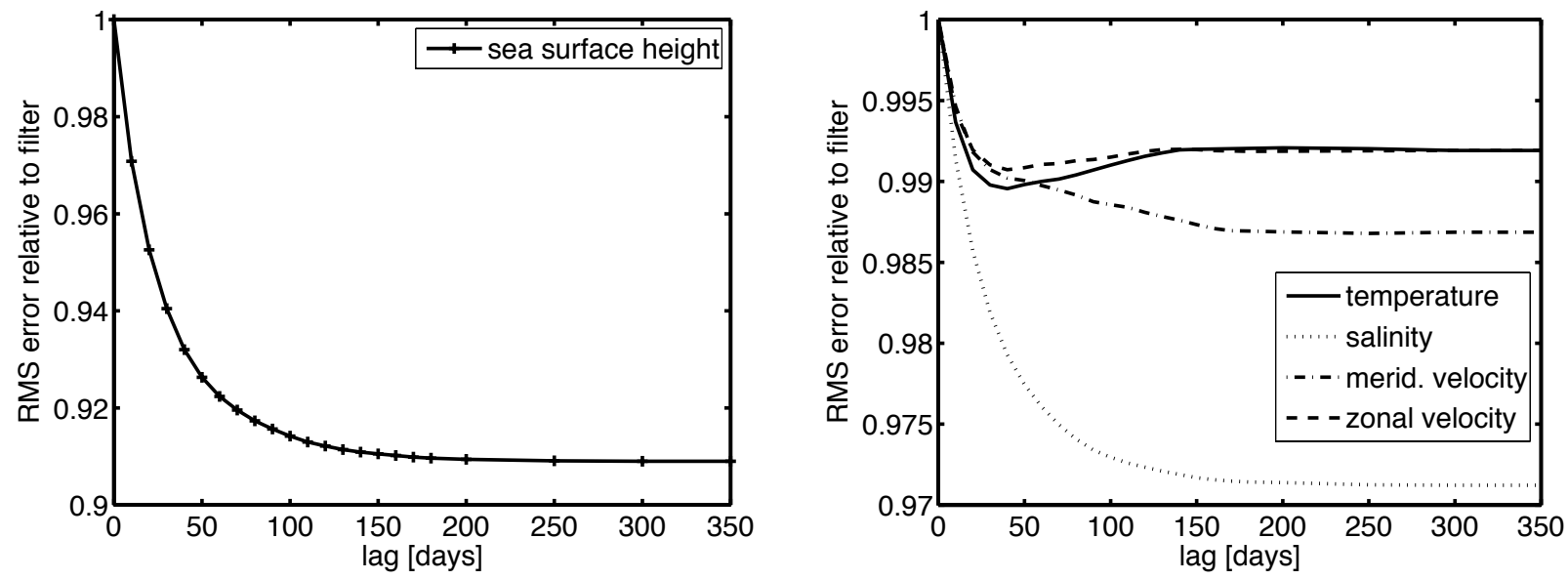

Figure 6. RMS errors relative to the RMS error obtained with the filter for different lags for the assimilation of pseudo SSH data. (left) relative RMS error for the sea surface height; (right) relative RMS errors for unobserved fields. 